\title{
Validation and cross-cultural adaptation of the Van Lieshout test in an Italian population with cervical spinal cord injury: a psychometric study
}

\author{
Giovanni Galeoto $\mathbb{D}^{1} \cdot$ Anna Berardi ${ }^{2} \cdot$ Rita De Santis ${ }^{3}$ Laura Di Valentini ${ }^{2} \cdot$ Rosmara Beccasio ${ }^{4}$. \\ Maria Auxiliadora Marquez ${ }^{5} \cdot$ Maria Luisa Giordano $^{6} \cdot$ Domenico Guarino $^{7} \cdot$ Marco Tofani $^{8}{ }^{8}$
}

Received: 6 April 2018 / Revised: 26 April 2018 / Accepted: 1 May 2018

(c) International Spinal Cord Society 2018

\begin{abstract}
Study design Psychometric study.

Objective To assess the reliability and validity of the Italian-translated and adapted version of the Van Lieshout test (VLT) in a population of people with cervical spinal cord injury (C-SCI).

Setting Multicenter study in multiple hospitals in Italy.

Methods The original scale was translated from English to Italian according to international guidelines. The internal consistency, test-retest reliability, and responsiveness of the Italian version of the VLT (IT-VLT) were examined. The ITVLT was administered to 50 individuals with C-SCI, and its construct and convergent validity were evaluated using Pearson correlation coefficients with the Italian version of the Spinal Cord Injury Measure version III (SCIM III).

Results All psychometric properties of the IT-VLT showed significant values, including the Cronbach's $\alpha$, which was 0.95 (left hand) and 0.94 (right hand). Furthermore, the test-retest reliability showed an intraclass correlation coefficient of 0.89 , 0.81 , and 0.87 for the right hand, left hand, and total value, respectively. The Pearson correlation coefficient of the IT-VLT and the Italian version of the SCIM III showed all significant results $r=0.559(p<0.01)$. The IT-VLT score improved significantly during in-patient rehabilitation In fact the mean $(95 \% \mathrm{CI})$ change between T0 and T1 was $-13.2 \pm 28.6$ ($21.38 ;-5.10)$.

Conclusions The IT-VLT was a reliable and a valid outcome measure for assessing hand function in the Italian population with C-SCI. The present study lays the grounds to investigate the psychometric properties of the short version of the ITVLT.
\end{abstract}

Giovanni Galeoto

giovanni.galeoto@uniroma1.it

1 Department of Public Health, Sapienza University of Rome, Rome, Italy

2 Sapienza" University of Rome, Rome, Italy

3 Department of Anatomical, Histological, Forensic and Orthopaedic Sciences, "Sapienza" University of Rome, Rome, Italy

4 "Cattolica" University of Rome, Rome, Italy

5 Occupational Therapist of Paraplegic Center Ostia, Ostia, Italy

6 Occupational Therapist of Orthopedic Trauma Center, Rome, Italy

7 Occupational therapist, Montecatone Rehabilitation Institute S.P. A, Imola, Italy

8 Department of Neurosciences and Neurorehabilitation Bambino Gesù Children's Hospital, Rome, Italy

\section{Introduction}

Tetraplegia results in varying degrees of functional losses in the neck, trunk, and upper and lower limbs depending on the severity and level of injury [1]. One of the most devastating aspects of a spinal cord injury (SCI) at the cervical level is the impairment of arm and hand function which greatly impacts level of independence and quality of life $[2,3]$. Restoration of arm and hand function is a specific priority for individuals with tetraplegia [4], and it is an important goal in the rehabilitation of cervical SCI (C-SCI).

In the literature, there are a number of assessment tools for the upper extremities, the most widely used are the Action Research Arm Test [5], the Jebsen-Taylor Hand Function Test [6], and the Disability of the Arm, Shoulder, and Hand [7]. The specific assessment tools for hand function in C-SCI individuals are the Tetraplegia Hand 
Activity Questionnaire (THAQ) [8], the Sollerman hand function test [9], the Graded Redefined Assessment of Strength, Sensibility and Prehension (GRASSP) [10], and the Van Lieshout test (VLT) [11]. An advantage of the VLT compared with the other tests is that it provides detailed information about how the tasks of the test are to be carried out, which might make it more useful in establishing treatment goals.

The VLT was developed in 2004 by Van Lieshout and colleagues as a specific tool to assess hand function in $\mathrm{C}$ SCI. The measurement concept of the VLT [11] refers to the International Classification of Functioning, Disability, and Health [12] and Nagi's Disability Model [13]. The VLT is composed of 19 tasks and it makes a distinction between basic and complex activities. The possible ways of performing each task are described in six hierarchical levels, resulting in a score from 0 to 5 . This tool is responsive to changes in arm/hand skilled performance during rehabilitation in people with C-SCI. The VLT was developed in the Dutch language. However, English and German versions are available from the author. In recent years, the VLT has been frequently considered as one of the most valuable tools for assessing hand function in C-SCI [14-17]. In 2006, Post et al. developed a short version of the VLT (VLT-SV) [18] to have a more useful instrument for research purposes. The VLT-SV includes 10 of the 19 tasks of the original VLT, and the level of performance of each task is scored within a range from 0 (worst arm/hand function) to 5 (best arm/hand function). The administration time is $25-35$ minutes. The VLT-SV has excellent internal consistency (Cronbach's alpha: 0.91) and inter-rater reliability (intraclass correlation coefficient (ICC) : 0.98 and 0.99), and very good convergent validity (Spearman correlation: 0.87-0.90).

In Italy, two assessment tools are typically used to assess hand function: the Jebsen-Taylor Hand Function test $[19,20]$ and the Disability of the Arm, Shoulder, and Hand [21] However, these are not specific for C-SCI and do not provide the necessary information to deal with the complexity of SCI. Thus, since 2014, the Italian National Coordination of Professional Operators in Spinal Units in the functional hand rehabilitation's protocol has indicated the VLT as one of the reference tool [22]. The VLT has also been indicated as a reference outcome measure in the Italian medicine, physics, and rehabilitation's guidelines [23]. The VLT was chosen for this study because, compared with the other tests, it provides detailed information about how the tasks of the test should be carried out, which might make it more useful in establishing treatment goals [24].

There are no psychometric studies of the VLT. This is surprising considering that the VLT is a starting point for hand function therapy and a tool to assist in clinical decision making about appropriate treatments. The aim of the present study was to not only translate and culturally adapt the VLT into Italian but also to investigate its psychometric properties in a population with C-SCI.

\section{Methods}

After receiving permission from the developers of the original instrument, the VLT was translated from English to Italian using the Translation and Cultural Adaptation of Patient-Reported Outcomes Measures-Principles of Good Practice guideline [25].

\section{Translation and cultural adaptation}

The original version of the VLT was translated into Italian by a panel of two native English speakers who produced two independent translations. An independent native speaker of the target language synthesized the results of the translations. Working from the temporary version of the VLT, two Italian translators then translated the questionnaire back into the original language without having seen the original version. The back-translated version of the instrument was then compared with the original. In order to adapt the translated version to Italian culture, two Italian rehabilitation professionals (an occupational therapist and a physiotherapist), who were familiar with both English and Italian, reviewed the first translated version and then reworded and reformulated some items to minimize any differences from the original version.

To evaluate its cross-cultural validity, the pre-final version of the Italian VLT (IT-VLT) was preliminarily applied to 10 participants that matched the target population. To ensure that the translation was comprehensible to the general or target population, participants' comments were recorded highlighting any items that could be inappropriate at a conceptual level and to identify any other issues that could potentially cause confusion. This resulted in the final version of the IT-VLT form being applied to the whole population of the study.

\section{Subjects and procedures}

Three Italian rehabilitation centers that specialized in the rehabilitation of people with SCIs participated in the study. The sample inclusion criteria were:

- A, B, C, or D level on the American Spinal Cord Injury Association Impairment Scale (AIS) [26];

- C-SCI (C3-T1 neurological level of injury);

- 18-65 years of age; and

- the ability to sit in a wheelchair for at least 3 consecutive hours. 
Exclusion criteria were C-SCI with severe additional neurological, orthopedic, or other diseases that interfered with the activities of daily living. In each participating rehabilitation center, one trained occupational therapist conducted the measurements according to a standardized protocol described in the research program. According to the original validation study [11], participants were assessed at the start of their functional rehabilitation program (T0), defined as the moment participants were just able to sit in their wheelchair for at least 3 consecutive hours; and 3 months later (T1) [27, 28].

\section{Assessment tools}

For the assessment of the participants, the Italian version of the Spinal Cord Injury Measure version III (SCIM III) [29, 30] was used. The SCIM III is a scale that rates the independence of patients with SCIs with regard to performing activities of daily living. Its comprises items on 19 daily tasks that are grouped into three subscales that assess: selfcare, respiration and sphincter management, and Mobility. The total SCIM III score ranges from 0 to 100, and higher scores reflect better performance or greater independence of a person.

\section{Data analysis}

The reliability and validity of the culturally adapted scale were assessed following the "Consensus-Based Standards for the Selection of Health Status Measurement Instruments" (COSMIN) checklist [31]. The ICC was used to assess the test-retest reliability. One therapist evaluated the same participant twice in a range of 7-10 days. An ICC value of $\geq 0.70$ was considered optimal to establish the degree to which repeated measurements were free from measurement error [32]. The internal consistency was used to assess the homogeneity of the scale and the interrelatedness of the items; a Cronbach's $\alpha$ coefficient of at least 0.70 was considered acceptable [33]. Pearson's correlation coefficient was used to evaluate construct and convergent validity. In the original study, the GraspRelease Test (GRT) [10] and the Jebsen-Tylor Hand Function Test [6] were used as the gold standard. Considering that the GRT has not been validated for the Italian population, and that the Jebsen-Taylor Hand Function Test was been validated after the start of the study $[19,20]$ for the present study, the Italian version of the SCIM III [30] was used to determine validity. To measure the responsiveness of the IT-VLT between $\mathrm{T} 0$ and $\mathrm{T} 1$, a pairedsamples $t$ test was used. A $p$ value of $<0.05$ was considered significant.

\section{Results}

\section{Translation and cultural adaptation}

After the translation process and the consensus of the expert panel, the translated version of the IT-VLT was formed. Cross-cultural validity was evaluated on 10 participants that matched the target population, these participants reported their observations in terms of comprehensibility and cognitive equivalence of the translation. Based on the results obtained from the preliminary application and the analysis of the report, it was possible to draw up the final version of the IT-VLT. No items were modified to improve comprehensibility and applicability, and all items were either identical or similar in meaning to the original version's items (see Appendix 1).

\section{Participants}

Individuals with C-SCI were recruited from December 2015 to December 2016 from the Spinal Cord Unites Centro Paraplegici Ostia and Centro Traumatologico Ortopedico Andrea Alesini in Rome, and Santa Maria della Misericordia Hospital in Perugia. The sample included $50 \mathrm{C}$ SCI individuals (22\% females and $78 \%$ males) with a mean age of $48( \pm 18)$ years. The characteristics of the sample are summarized in Table 1.

\section{Data analysis}

The mean score for the total VLT scale was $75 \pm 49$ and there were no ceiling or floor effects. The Cronbach's $\alpha$ for the

Table 1 Demographic characteristics for the 50 participants in the reliability study of the IT-VLT

\begin{tabular}{lc}
\hline Age $($ mean \pm SD) & $47.7 \pm 17.5$ \\
\hline Gender men $(\%)$ & 78 \\
Neurological level of injury number (\%) \\
C2 \\
C3 & $1(2)$ \\
C4 & $1(2)$ \\
C5 & $15(30)$ \\
C6 & $10(20)$ \\
C7 & $15(30)$ \\
American spinal injury association impairment scale (AIS) number \\
$(\%)$ & $3(6)$ \\
A & $19(38)$ \\
B & $12(24)$ \\
C & $14(28)$ \\
D & $5(10)$ \\
\hline
\end{tabular}


Table 2 Item-total analysis: Cronbach's alpha value for all the items on 50 participants

\begin{tabular}{|c|c|c|c|c|c|c|c|c|}
\hline & \multicolumn{4}{|c|}{ VLT left hand } & \multicolumn{4}{|c|}{ VLT right hand } \\
\hline & $\begin{array}{l}\text { Scale } \\
\text { mean if } \\
\text { item } \\
\text { deleted }\end{array}$ & $\begin{array}{l}\text { Scale } \\
\text { variance } \\
\text { if item } \\
\text { deleted }\end{array}$ & $\begin{array}{l}\text { Corrected } \\
\text { item-total } \\
\text { correlation }\end{array}$ & $\begin{array}{l}\text { Cronbach's } \\
\text { alpha if item } \\
\text { deleted }\end{array}$ & $\begin{array}{l}\text { Scale } \\
\text { mean if } \\
\text { item } \\
\text { deleted }\end{array}$ & $\begin{array}{l}\text { Scale } \\
\text { variance } \\
\text { if item } \\
\text { deleted }\end{array}$ & $\begin{array}{l}\text { Corrected } \\
\text { item-total } \\
\text { correlation }\end{array}$ & $\begin{array}{l}\text { Cronbach's } \\
\text { alpha if item } \\
\text { deleted }\end{array}$ \\
\hline ITEM1 & 34.14 & 588.57 & 0.37 & 0.95 & 36.44 & 611.72 & 0.48 & 0.95 \\
\hline ITEM2 & 34.84 & 590.63 & 0.42 & 0.94 & 37.14 & 618.37 & 0.49 & 0.95 \\
\hline ITEM3 & 35.02 & 570.67 & 0.51 & 0.94 & 37.32 & 594.83 & 0.60 & 0.95 \\
\hline ITEM4 & 34.48 & 548.01 & 0.70 & 0.94 & 36.78 & 579.40 & 0.71 & 0.95 \\
\hline ITEM5 & 34.54 & 546.42 & 0.78 & 0.94 & 36.94 & 579.40 & 0.75 & 0.95 \\
\hline ITEM6 & 34.76 & 552.39 & 0.80 & 0.94 & 36.82 & 578.56 & 0.82 & 0.94 \\
\hline ITEM7 & 35.24 & 567.78 & 0.67 & 0.94 & 37.40 & 600.37 & 0.66 & 0.95 \\
\hline ITEM8 & 34.90 & 549.03 & 0.77 & 0.94 & 36.94 & 577.24 & 0.78 & 0.94 \\
\hline ITEM9 & 33.40 & 564.45 & 0.60 & 0.94 & 35.26 & 598.40 & 0.66 & 0.95 \\
\hline ITEM10 & 34.26 & 576.03 & 0.63 & 0.94 & 36.56 & 621.93 & 0.48 & 0.95 \\
\hline ITEM11 & 34.72 & 563.19 & 0.64 & 0.94 & 36.92 & 599.10 & 0.61 & 0.95 \\
\hline ITEM12 & 34.96 & 553.79 & 0.79 & 0.94 & 36.74 & 586.85 & 0.77 & 0.95 \\
\hline ITEM13 & 34.78 & 559.40 & 0.79 & 0.94 & 36.90 & 586.13 & 0.83 & 0.94 \\
\hline ITEM14 & 34.62 & 573.179 & 0.63 & 0.94 & 36.88 & 613.90 & 0.53 & 0.95 \\
\hline ITEM15 & 34.66 & 561.00 & 0.71 & 0.94 & 36.68 & 587.37 & 0.79 & 0.94 \\
\hline ITEM16 & 34.76 & 557.25 & 0.73 & 0.94 & 36.86 & 583.76 & 0.78 & 0.94 \\
\hline ITEM17 & 34.58 & 569.56 & 0.67 & 0.94 & 36.88 & 593.74 & .78 & .95 \\
\hline ITEM18 & 34.70 & 560.75 & 0.69 & 0.94 & 37.00 & 592.61 & .69 & .95 \\
\hline ITEM19 & 35.08 & 559.83 & 0.78 & 0.94 & 37.38 & 590.56 & .80 & .94 \\
\hline TOTAL & & & & 0.95 & & & & 0.94 \\
\hline
\end{tabular}

Table 3 Test-retest analysis: range of ICC parameters of left and right hand on 35 participants

\begin{tabular}{lllll}
\hline & Test mean \pm SD & Retest mean \pm SD & ICC & IC 95\% \\
\hline VLT left & $34.67 \pm 24.22$ & $39.11 \pm 23.55$ & 0.81 & $0.76-0.96$ \\
VLT right & $38.78 \pm 25.88$ & $44.33 \pm 24.57$ & 0.90 & $0.73-0.98$ \\
VLT total & $73.44 \pm 48.96$ & $83.44 \pm 46.98$ & 0.88 & $0.87-0.97$ \\
\hline
\end{tabular}

IT-VLT was 0.95 and $0.94(p<0.01)$ for the left and right hand, respectively. Internal consistency was calculated for the entire scale; the item-total correlation showed positive results as reported in Table 2 . The test-retest reliability was evaluated on 35 participants with chronic C-SCI. The ITVLT showed an ICC of $0.89,0.81$, and 0.87 for the right hand, left hand, and total value, respectively. ICC values are reported in Table 3. The Pearson's correlation coefficient of the IT-VLT and the SCIM III were significant $(p<0.01$ for all). IT-VLT correlates also with the neurological level of injury but not with the AIS [26]. All the results are summarized in Table 4).

The responsiveness was evaluated on the overall sample. The null hypothesis that no clinical change would occur between $\mathrm{T} 0$ and $\mathrm{T} 1$ was rejected, as shown in Table 5. In fact the mean $(95 \% \mathrm{CI})$ change between $\mathrm{T} 0$ and $\mathrm{T} 1$ was $13.2(-21.38 ;-5.10)$.

\section{Discussion}

The objective of this study was to translate and culturally adapt the VLT for the Italian culture and investigate its psychometric properties in a sample of people with C-SCI. The translation process and cross-cultural adaptation, following international guidelines [25], allowed us to compile the IT-VLT manual. The Italian version of the manual will be available in accordance with the copyright owner's royalties (www.adelante-zorggroep.nl). The participants involved showed homogeneous characteristics, and the findings of the present study demonstrated good psychometric properties of the assessment tool. Considering that the VLT was formed for clinical purposes, the responsiveness was a fundamental criterion to analyze, the IT-VLT showed a high rate of measured change over time in the hand functions of C-SCI individuals $(-13.2 \mathrm{CI}=-21.38$; $-5.10)$. In literature there are no data on the psychometric properties of the VLT so the research team wanted to investigate its validity and reliability. The IT-VLT showed excellent internal consistency (left hand $=0.95$, right hand $=0.94)$. In addition, all ICC were $>0.70$, thus demonstrating good reliability of the scale. The Pearson's correlation coefficient of the IT-VLT and the Italian version of the SCIM III [30] were significant $(p<0.01)$, indicating that 
Table 4 Construct and convergent validity analysis: Pearson's correlation between IT-VLT and the Italian version of the SCIM III, AIS, and neurological level of injury

Table $5 t$ test analysis: responsiveness of the IT-VLT

\begin{tabular}{llll}
\hline VLT & Pearson $r$ SCIM III & Pearson $r$ AIS scale & Pearson $r$ neurological level of injury \\
\hline VLT left & $0.53^{*}$ & 0.18 & $0.64^{*}$ \\
VLT right & $0.55^{*}$ & 0.11 & $0.69^{*}$ \\
VLT total & $0.56^{*}$ & 0.15 & $0.68^{*}$ \\
$* P<0.01$ & & & \\
\hline
\end{tabular}

\begin{tabular}{lcrll}
\hline & T0 mean \pm SD & T1 mean \pm SD & Mean difference \pm SD $($ CI 95\% $)$ & $p$ \\
\hline VLT left & $36.58 \pm 25.02$ & $43.36 \pm 27.06$ & $-6.7 \pm 14.8(-10.98 ;-2.58$ & 0.002 \\
VLT right & $38.88 \pm 25.69$ & $45.34 \pm 27.20$ & $-6.5 \pm 14.8(-10.68 ;-2.24)$ & 0.003 \\
VLT total & $75.46 \pm 49.19$ & $88.7 \pm 52.53$ & $-13.2 \pm 28.6(-21.38 ;-5.10)$ & 0.002 \\
\hline
\end{tabular}

higher hand function in individuals with C-SCI reflects better performance or greater independence in everyday life. These findings are consistent with the VLT-SV [18] study, which showed that the IT-VLT is correlated with the neurological level of injury. Based on our findings, it is possible to state that the IT-VLT is a valid and reliable measure for assessing hand function in C-SCI individuals, the result suggest that it could be used as a starting point for hand function therapy, as a predictor to future independence in activity of daily living and then to assist in clinical decision making as to the treatment policy. Finally, it could be used as an outcome measure in randomized controlled trials.

Nevertheless, this study has some limitations. First, the relatively small sample size did not allow an understanding of the differences between people with different levels of CSCI. Furthermore, a larger group of participants would probably have given a better impression of the relationship between arm/hand impairment and the VLT score. Another limitation is that is it not possible to compare our data with other studies, because in the literature, there are no psychometric studies of the VLT. Moreover, these studies report only the value of the hand that obtained the best performance, so all attempts to compare data are fraught with difficulties. Future studies are needed to extend the sample size allowing a better understanding of the relationship between the VLT score and different levels of CSCI. Furthermore, further work is needed to examine interrater reliability and concurrent validity with the JebsenTaylor Hand Function Test now available in its Italian valid and reliable version [19, 20]. This work also lays the basis for developing and investigating the psychometric properties of the short version of the IT-VLT.

In conclusion, the present study gives Italian health professionals and researchers a sensitive and valid tool to investigate and describe hand function of people with tetraplegia in a reproducible way. Given the great usefulness of this test in measuring hand function in C-SCI individuals, we hope that our study is a starting point and that it encourages rehabilitators worldwide to validate the test in their own cultural context.

Acknowledgements We acknowledge those who participated in the surveys; their collective expertize greatly informed the development of the IT-VLT.

\section{Compliance with ethical standards}

Conflict of interest The authors declare that they have no conflict of interest.

Statement of ethics We certify that all applicable institutional and governmental regulations concerning the ethical use of human volunteers were followed during the course of this research.

Statement of human and animal rights All procedures followed were in accordance with the ethical standards of the responsible committee on human experimentation (institutional andnational) and with the Helsinki Declaration of 1975, as revised in 2008. Informed consent was obtained from all participants for being included in the study.

\section{References}

1. Biering-Sørensen F, Bickenbach JE, El Masry WS, Officer A, von Groote PM. ISCoS-WHO collaboration. International Perspectives of Spinal Cord Injury (IPSCI) report. Spinal Cord. 2011;49:679-83.

2. Snoek GJ, IJzerman MJ, Hermens HJ, Maxwell D, BieringSorensen F. Survey of the needs of patients with spinal cord injury: impact and priority for improvement in hand function in tetraplegics. Spinal Cord. 2004;42:526-32.

3. Simpson LA, Eng JJ, Hsieh JT, Wolfe DL. The health and life priorities of individuals with spinal cord injury: a systematic review. J Neurotrauma. 2012;29:1548-55.

4. Franke AC, Snoek GJ, de Groot S, Nene AV, Spooren AI, Post MW. Arm hand skilled performance in persons with a cervical spinal cord injury--long-term follow-up. Spinal Cord. 2013;51:161-4.

5. Hsieh CL, Hsueh IP, Chiang FM, Lin PH. Inter-rater reliability and validity of the action research arm test in stroke patients. Age Ageing. 1998;27:107-13.

6. Jebsen RH, Taylor N, Trieschmann RB, Trotter MJ, Howard LA. An objective and standardized test of hand function. Arch Phys Med Rehabil. 1969;50:311-9. 
7. Hudak PL, Amadio PC, Bombardier C. Development of an upper extremity outcome measure: the DASH. Am J Ind Med. 1996;29:602-8.

8. Land NE, Odding E, Duivenvoorden HJ, Bergen MP, Stam HJ. Tetraplegia Hand Activity Questionnaire (THAQ): the development, assessment of arm-hand function-related activities in tetraplegic patients with a spinal cord injury. Spinal Cord. 2004;42:294-301.

9. Sollerman C, Ejeskär A. Sollerman hand function test. A standardised method and its use in tetraplegic patients. Scand J Plast Reconstr Surg Hand Surg. 1995;29:167-76.

10. Kalsi-Ryan S, Curt A, Verrier MC, Fehlings MG. Development of the Graded Redefined Assessment of Strength, Sensibility and Prehension (GRASSP): reviewing measurement specific to the upper limb in tetraplegia. J Neurosurg Spine. 2012;17:65-76.

11. Van Lieshout, G. User manual Van Lieshout Test. (2003).

12. World Health Organization. International Classification of Functioning, Disability and Health. Geneva: World Health Organization; 2001.

13. Nagi SZ. A study in the evaluation of disability and rehabilitation potential: concepts, methods, and procedures. Am J Public Health Nations Health. 1964;54:1568-79.

14. Spooren AI, Janssen-Potten YJ, Post MW, Kerckhofs E, Nene A, Seelen HA. Measuring change in arm hand skilled performance in persons with a cervical spinal cord injury: responsiveness of the Van Lieshout Test. Spinal Cord. 2006;44:772-9.

15. Reinkensmeyer DJ, Dietz V. Neurorehabilitation Technology. 2nd edn., Zurich, Switzerland: Springer; 2011.

16. Cardenas D. Spinal Cord Medicine. 2nd edn., New York: Principles \& Practice; 2012.

17. Duruoz MT. Hand Function: A Practical Guide to Assessment. Zurich, Switzerland: Springer; 2014.

18. Post MW, Van Lieshout G, Seelen HA, Snoek GJ, Ijzerman MJ, Pons C. Measurement properties of the short version of the Van Lieshout test for arm/hand function of persons with tetraplegia after spinal cord injury. Spinal Cord. 2006;44:763-71.

19. Culicchia G, Nobilia M, Asturi M, Santilli V, Paoloni M, De Santis $\mathrm{R}$, et al. Cross-cultural adaptation and validation of the Jebsen-Taylor hand function test in an Italian population. Rehabil Res Pract. 2016;2016:8970917.

20. Nobilia M, Culicchia G, Guarino D, De Santis R, Savona A, Tofani M, et al. The Italian version of the Jebsen-Taylor Hand Function Test for the evaluation of hand disorders: a crosssectional study. Am J Occup Ther, in press.
21. Padua R, Padua L, Ceccarelli E, Romanini E, Zanoli G, Amadio PC, et al. Italian version of the Disability of the Arm, Shoulder and Hand (DASH) questionnaire. Cross-cultural adaptation and validation. J Hand Surg. 2003;28:179-86.

22. Il protocollo della mano funzionale - CNOPUS. [online] Available at: http://www.cnopus.it/il-protocollo-della-mano-funzionale/.

23. Formazionesostenibile.it. (2018). [online] Available at: http://www.formazionesostenibile.it/2017/RomeRehabilitation/ libro_per_sito.pdf

24. Van Tuijl JH, Janssen-Potten YJ, Seelen HA. Evaluation of upper extremity motor function tests in tetraplegics. Spinal Cord. 2002;40:51-64.

25. Wild D, Grove A, Martin M, Eremenco S, McElroy S, VerjeeLorenz A, et al. Principles of good practice for the translation and cultural adaptation process for patient-reported outcomes (PRO) measures: report of the ISPOR task force for translation and cultural adaptation. Value Health. 2005;8:94-104.

26. American Spinal Injury Association/International Medical Society of Paraplegia. International Standards for Neurological and Functional Classification of Spinal Cord Injury Patients. Chicago: American Spinal Injury Association; 2000.

27. Galeoto G, De Santis R, Marcolini A, Cinelli A, Cecchi R. Il consenso informato in Terapia Occupazionale: proposta di una modulistica. G Ital Med Lav Ergon. 2016;38:107-15.

28. Galeoto G, Mollica R, Astorino O, Cecchi R. Informed consent in physiotherapy: proposal of a form. G Ital Med Lav Ergon. 2015;37:245-54.

29. Catz A, Itzkovich M, Agranov E, Ring H, Tamir A. SCIM-spinal cord independence measure: a new disability scale for patients with spinal cord lesions. Spinal Cord. 1997;35:850-6.

30. Invernizzi M, Carda S, Milani P, Mattana F, Fletzer D, Iolascon $\mathrm{G}$, et al. Development and validation of the Italian version of the Spinal Cord Independence Measure III. Disabil Rehabil. 2010;32:1194-203.

31. Mokkink LB, Terwee CB, Patrick DL, Alonso J, Stratford PW, Knol DL, et al. The COSMIN checklist for assessing the methodological quality of studies on measurement properties of health status measurement instruments: an international Delphi study. Qual Life Res. 2010;19:539-49.

32. Cicchetti DV. Guidelines, criteria, and rules of thumb for evaluating normed and standardized assessment instruments in psychology. Psychol Assess. 2014;6:284-90.

33. Nunnally JC. Psychometric theory. New York: McGraw-Hill; 1978. 